\title{
A little less conversation, a little more high impact action
}

\author{
Authors: Zoë Burton* and Helena Edwards
}

\section{Aims}

NHS Improvement's 'Eight high impact actions to improve

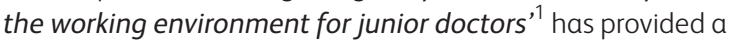
framework for the majority of the improvement initiatives we have implemented as chief registrars (CRs) at Portsmouth Hospitals NHS Trust. Additional projects have largely centred around improving patient safety and junior doctor (JD) morale.

\section{Methods}

A survey was conducted in August 2017 to identify issues threatening doctors' efficiency and enjoyment in their clinical roles. A monthly JD Forum was also initiated giving JDs a regular opportunity to raise issues or concerns and present their ideas for innovation. Ideas were subsequently developed and presented at executive level under the umbrella of the 'Eight high impact actions'. Each action has been progressed separately and this is reflected by varying degrees of completion.

\section{Results}

Ninety-eight cross-specialty survey respondents included all JD grades. Suggestions for key improvements and efficiency savings to be made across the trust ranged from better information technology solutions, printed blood bottle labels, bleep-free communication and improved access to clinical guidelines, to seeing the chief executive 'on the shop floor' and a better dialogue between management and front-line staff. $62.2 \%$ stated they had difficulty accessing trust clinical guidelines and $81.6 \%$ believed a smartphone app would be beneficial.

Suggestions for further quality improvement (QI) initiatives arose directly from JD forums at later dates. Table 1 illustrates the key improvement projects that we have initiated or supported. The JD Forum, 'paired learning' and 'lessons learnt' programmes have been particularly successful with regular attendance and positive feedback. While the introduction of the clinical guidelines smartphone app has encountered multiple setbacks, an app platform has been funded, a governance system agreed, and a QI package designed to support JDs in reformatting guidelines prior to app upload.

\section{Conclusion}

During our time as CRs, we have received excellent support from senior management which has helped pave the way in initiating changes in

Author: Portsmouth Hospitals NHS Trust, Portsmouth, UK *Royal College of Physicians chief registrar

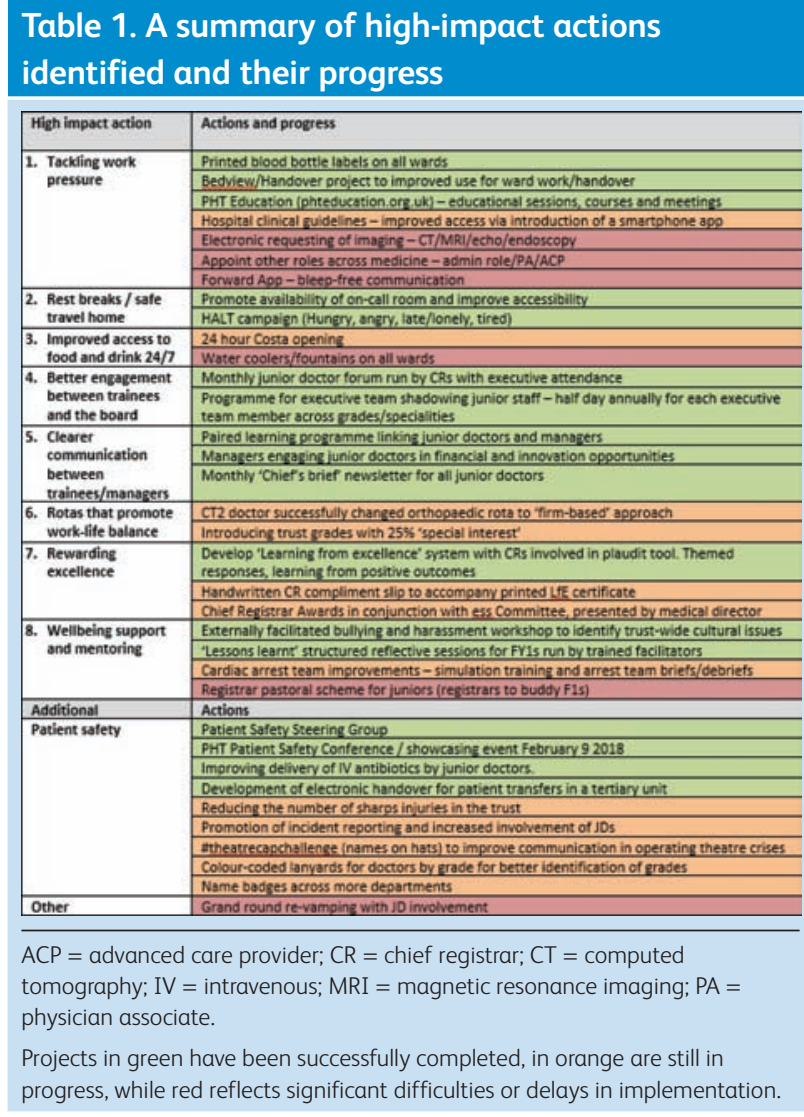

practice and culture. Remaining $50 \%$ in clinical practice has enabled us to understand the complexity of difficulties encountered by both JDs and senior management. One of the most valuable aspects of our role has been to give JDs a voice by providing a two-way clinicalmanagerial communication channel. We have taken on a large number of projects, some of which are difficult to measure or gauge success. Despite our efforts, other projects remain in their infancy or will take time to come to fruition, highlighting the difficulties we have encountered.

\section{Conflict of interest statement}

None.

\section{Reference}

1 NHS Improvement. Eight high impact actions to improve the working environment for junior doctors. NHS, 2017. 